\title{
A comparison of antibiotic prescription by doctors and dentists for acute dental conditions
}

\author{
Antibiotic prescribing for dental conditions: general medical practitioners and dentists compared $R$. Anderson, \\ L. Calder, and D. W. Thomas Br Dent J 2000; 188: 398-400
}

\author{
Objective \\ To compare the level of prescribing and types of antibiotics \\ prescribed for dental problems by general medical practitioners \\ and dentists.
}

\section{Design}

Secondary analysis of standard consultation data and prescription records from four different settings.

\section{Setting}

30 participating general practices in the General Practice Morbidity Database for Wales in 1996.

\section{Subjects \\ 1,185 attendances for tooth-related problems, at 30 participating practices in the General Practice Morbidity Database for Wales in 1996. Standard consultation records from a GDP emergency dental rota, and two weekend emergency dental clinics: one in a health centre, the other in a dental hospital.}

\section{Results}

More than two thirds (68\%) of attendances at general medical practices for tooth-related problems resulted in a prescription for antibiotics. In contrast less than a third $(28 \%)$ of patients seen by a GDP rota, about half (52\%) of patients at a weekend emergency clinic in a health centre, and just more than a third (38\%) of patients attending the dental hospital clinic received antibiotics. General medical practitioners were also more likely to prescribe broad-spectrum antibiotics than dentists.

\section{Conclusion}

For acute dental problems general medical practitioners are more likely to prescribe antibiotics than dentists. There also appear to be inter-professional differences in the tendency to prescribe broad spectrum antibiotics. Initiatives to rationalise prescribing for dental conditions may need to target doctors as well as dentists.

\section{In brief}

- The appropriate prescription of antibiotics is of increasing concern for both doctors and dentists.

- This study reveals wide variations in the level of antibiotic prescribing for acute dental conditions in different primary care settings.

- Initiatives to rationalise prescribing for dental conditions may need to target doctors as well as dentists.

\section{Comment}

The problem of antibiotic resistance has 1 highlighted the need for rational prescribing. This interesting study compares the prescribing of antibiotics by general medical practitioners (GMPs) and dentists for dental emergencies. The authors admit that the data from all the four sources sampled could not be directly compared. It is difficult to determine, for example, whether the patients presenting with dental problems and receiving antibiotics really had acute infections.

The authors correctly state that antibiotics without dental treatment are ineffective in the management of acute dental problems. There is also no indication for antibiotics in the absence of infection. The importance of a comprehensive history, examination and accurate diagnosis before prescribing of antibiotics cannot be over- stressed. Most GMPs have not been trained in the diagnosis and treatment of dental problems. It was therefore not surprising, but still disconcerting, to find within this study a large proportion $(68 \%)$ of patients with acute dental problems received antibiotics from general medical practitioners. Fear, cost and the accessibility of dental treatment may play a part in patients attending their general medical practitioner, rather than a dentist, for treatment.

More than half of the patients attending a health authority emergency clinic received antibiotics. This may be caused by the large number of 'unregistered' patients attending where antibiotics are probably prescribed because of the pressure of time and workload. In contrast, only $28 \%$ of mainly registered patients received antibiotics when seen as emergencies at the surgeries of den- tists in an LDC organised rota. This study also showed that both general medical practitioners and dentists prefer to prescribe broad-spectrum antibiotics, contrary to the Dental Practitioners' Formulary recommendation of penicillin.

Dental care should be more accessible, minimising the need for patients to seek emergency dental treatment from general medical practitioners. The Government's proposed 'dental access centres' and NHS Direct might play a part in improving access, thereby reducing antibiotic prescribing for dental emergencies in the future.

\section{N. Palmer}

General Dental Practitioner, Crosby, Liverpool and Part-time Lecturer Clinical Dental Sciences Department, University of Liverpool 